
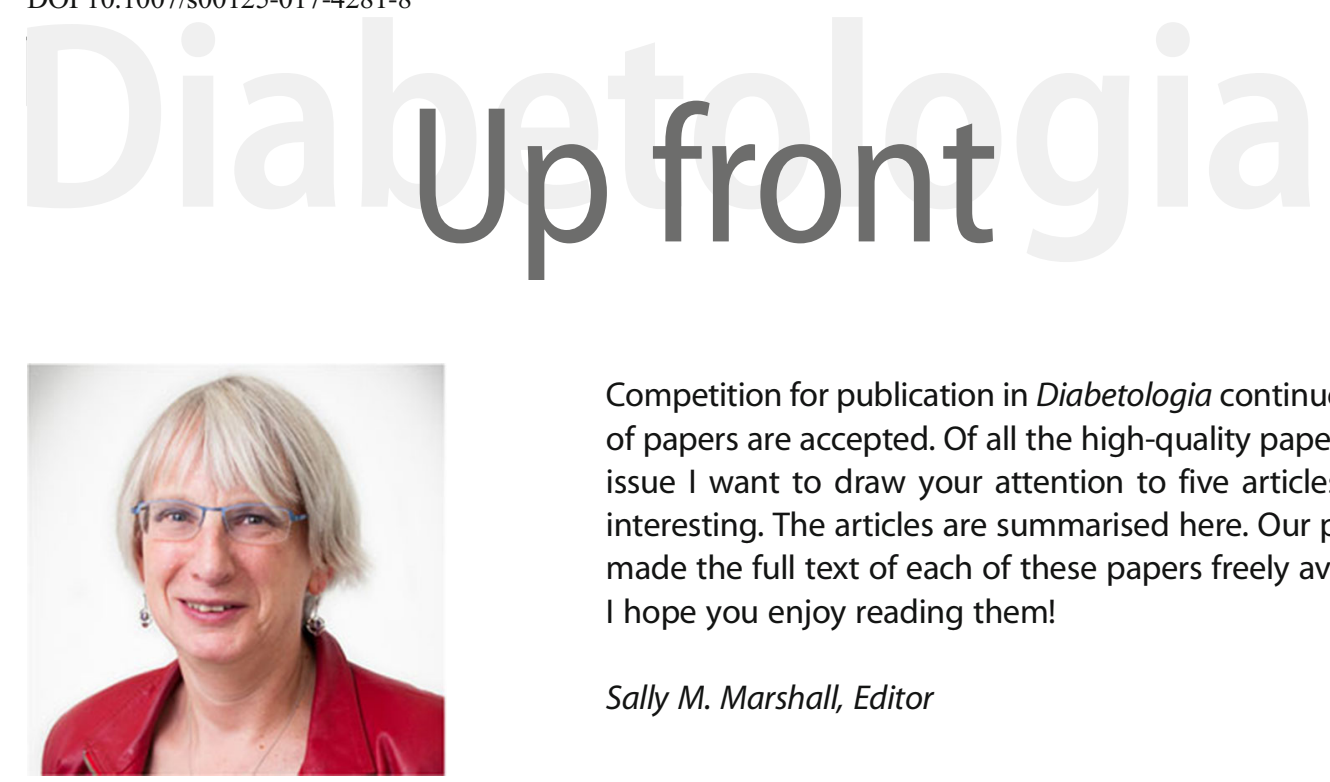

Competition for publication in Diabetologia continues to grow, and less than $20 \%$ of papers are accepted. Of all the high-quality papers that appear in this month's issue I want to draw your attention to five articles that I think are particularly interesting. The articles are summarised here. Our publisher, Springer, has kindly made the full text of each of these papers freely available.

I hope you enjoy reading them!

Sally M. Marshall, Editor

Published online: 26 April 2017

The gut microbiome as a target for prevention and treatment of hyperglycaemia in type 2 diabetes: from current human evidence to future possibilities

Louise Brunkwall, Marju Orho-Melander

The modifiable capacity of the gut microbiota is increasingly recognised as a promising target for improvements in glycaemic control and the treatment of type 2 diabetes. In this issue, Brunkwall and Orho-Melander (DOI 10.1007/ s00125-017-4278-3) review the current human evidence and future possibilities and challenges of using the gut microbiota in the development of preventive and treatment strategies for type 2 diabetes. Several potential gut-targeting glucose-lowering therapies have emerged and show initial promise, but a better understanding of their mechanisms of action in humans is required. So far, human gut microbiota studies have been limited in size and large longitudinal and interventional studies are required. Amongst the great challenges for the future are the personalisation of nutrition to allow for individual dietary advice (taking into account the gut microbiota, genetics, drug use and other relevant environmental factors) and the integration of multi-omic human and microbiota data in large, longitudinal cohorts. For now, however, the potential for gut microbiota in the prevention and management of diabetes remains a hype, but a hype with plenty of hope.
The islet endothelial cell: a novel contributor to beta cell secretory dysfunction in diabetes

Meghan F. Hogan, Rebecca L. Hull

The role of the islet endothelial cell in regulating islet beta cell growth and function under physiological conditions is now well established. An emerging literature demonstrates that islet microvascular abnormalities occur in type 2 diabetes and may contribute to the impaired beta cell function and survival that characterise this disease. In this issue, Hogan and Hull (DOI 10.1007/s00125-017-4272-9) review the current literature in this area. They review studies describing a number of endothelialderived factors that stimulate beta cell growth, insulin production and release. Then, data showing abnormal islet capillary morphology in human type 2 diabetes are summarised and some important differences between humans and animal models are noted. Mechanisms underlying the induction of islet endothelial dysfunction in diabetes, their functional impact and potential therapeutic strategies are also discussed. Finally, caution is urged in extrapolating the beneficial effects of targeting the expansion of islet vasculature under normal conditions to metabolic disease. 
Human genetics as a model for target validation: finding new therapies for diabetes

Soren K. Thomsen, Anna L. Gloyn

Conventional preclinical models have limited ability to predict safety and efficacy profiles of new treatments in humans. As a result, the vast majority of drug candidates fail to progress from clinical trials, driving the escalating costs of pharmaceutical research and development programmes. In this issue, Thomsen and Gloyn (DOI 10.1007/s00125-017-4270-y) review the role of human genetics as a complementary model for drug target validation. Naturally occurring genetic perturbations provide a window into causal human biology that can be used to predict both beneficial and adverse effects of drugs. The authors demonstrate how genetic discoveries over the past decades have confirmed drug mechanisms for existing type 2 diabetes treatments, including sulfonylureas, thiazolidinediones, glucokinase activators and sodium-glucose cotransporter 2 (SGLT2) inhibitors. To apply this strategy prospectively, experimental advances will be required to accelerate the translation of genetic findings into molecular mechanisms. Recent developments have provided promising solutions, paving the way for the future discovery of new diabetes therapies.

\section{Cognitive deficits associated with impaired awareness of hypoglycaemia in type 1 diabetes}

Tor I. Hansen, Sandra E. Olsen, Elise C. D. Haferstrom, Trond Sand, Brian M. Frier, Asta K. Håberg, Marit R. Bjørgaas

An association between impaired awareness of hypoglycaemia (IAH) and cognitive deficiency has previously been suggested but the putative role of impaired cognitive function in recurrent severe hypoglycaemia is unclear. In this issue, Hansen and colleagues (DOI 10.1007/s00125-017-4233-3) compare cognitive function in people with type 1 diabetes and established IAH with those in whom hypoglycaemia awareness is intact. They report that people with IAH had significant impairments in learning, memory and pattern separation, all of which rely on the integrity of the hippocampus. Participants followed standardised aural and written instructions and the frequency of severe hypoglycaemia correlated with the number of tests that had not been performed according to these instructions. The authors suggest that adults with IAH may have a reduced cognitive reserve, which may contribute to their increased risk of developing severe hypoglycaemia. Furthermore, the present results support a role for recurrent severe hypoglycaemia in the pathogenesis of IAH. These observations underline the value of including cognitive tests in intervention programmes to evaluate whether impaired cognitive ability may affect adherence to treatment and outcomes in type 1 diabetes.

Association between type 2 diabetes and risk of cancer mortality: a pooled analysis of over 771,000 individuals in the Asia Cohort Consortium

Yu Chen, Fen Wu, Eiko Saito, Yingsong Lin, Minkyo Song, Hung N. Luu, Prakash C. Gupta, Norie Sawada, Akiko Tamakoshi, Xiao-Ou Shu, Woon-Puay Koh, Yong-Bing Xiang, Yasutake Tomata, Kemmyo Sugiyama, Sue K. Park, Keitaro Matsuo, Chisato Nagata, Yumi Sugawara, You-Lin Qiao, San-Lin You, Renwei Wang, Myung-Hee Shin, Wen-Harn Pan, Mangesh S. Pednekar, Shoichiro Tsugane, Hui Cai, Jian-Min Yuan, Yu-Tang Gao, Ichiro Tsuji, Seiki Kanemura, Hidemi Ito, Keiko Wada, Yoon-Ok Ahn, Keun-Young Yoo, Habibul Ahsan, Kee Seng Chia, Paolo Boffetta, Wei Zheng, Manami Inoue, Daehee Kang, John D. Potter

Emerging evidence is suggestive of a link between type 2 diabetes and an increased risk of developing cancer and death from cancer. However, the majority of studies in this area have been conducted in Western populations. In this issue, Chen et al (DOI 10.1007/s00125-017-4229-z) report whether Asian individuals with type 2 diabetes were more likely to die from cancer, using data from 771,000 individuals from the Asia Cohort Consortium. The authors found that having type 2 diabetes led to a $26 \%$ increase in the risk of dying from any form of cancer in Asians. More specifically, type 2 diabetes was related to the risk of death from cancers of the colorectum, liver, bile duct, gallbladder, pancreas, breast, endometrium, ovary, prostate, kidney and thyroid, and lymphoma. The strongest associations were observed for cancers of the liver, thyroid and kidney (approximately double the risk in each case), endometrium (2.7 times increased risk) and breast (1.7 times increased risk). These data suggest that the influence of type 2 diabetes on the risk of death from cancer is largely similar in Asian individuals and those from developed Western countries and that type 2 diabetes should be considered a risk factor for cancers in Asians. The authors conclude that the pattern of associations between type 2 diabetes and specific cancers (particularly liver cancer, which has a high incidence in Asian individuals) suggests the need for better control of the growing epidemic of diabetes in order to reduce cancer mortality rates.

All text supplied by the authors. 